\title{
Coordinate System for Learning in the Smooth Pursuit Eye Movements of Monkeys
}

\author{
Maninder Kahlon and Stephen G. Lisberger \\ Department of Physiology, W. M. Keck Foundation Center for Integrative Neuroscience, and Neuroscience Graduate \\ Program, University of California, San Francisco, San Francisco, California 94143
}

\begin{abstract}
Learning was induced in smooth pursuit eye movements by repeated presentation of targets that moved at one speed for $100 \mathrm{msec}$ and then changed to a second, higher or lower, speed. The learned changes, measured as eye acceleration for the first 100 msec of pursuit, were largest in a "late" interval from 50 to $80 \mathrm{msec}$ after the onset of pursuit and were smaller and less consistent in the earliest $30 \mathrm{msec}$ of pursuit. In each experiment, target motion in one direction consisted of learning trials, whereas target motion in the opposite (control) direction consisted of trials in which targets moved at a constant speed for the entire duration of the trial. Under these conditions, the learning did not generalize to the control direction. For target motion in the learning direction, the changes in pursuit generalized to responses evoked by targets moving at speeds ranging from 15 to $45 \%$ sec as well as to targets of different colors and sizes. Although learning was induced at the initiation of
\end{abstract}

pursuit, it generalized to the response to image motion in the learning direction when it was presented during pursuit in the learning direction. However, learning did not generalize to the response to image motion in the learning direction when it was presented during pursuit in the control direction. The results suggest that the learning does not occur in purely sensory or motor coordinates but in an intermediate reference frame at least partly defined by the direction of eye movement. The selectivity of learning provides new evidence for a previously hypothesized neural "switch" that gates visual information on the basis of movement direction. This selectivity also suggests that the locus of pursuit learning is in pathways related to the operation of the switch.

Key words: motor learning; smooth pursuit eye movements; monkey; visual motion; sensory-motor transformation; coordinate system
Smooth pursuit is a voluntary eye tracking behavior that, because it is well characterized both behaviorally and neurally, provides an excellent opportunity for the analysis of learning in complex, voluntary motor systems. Pursuit allows primates to track a small target that is moving across a stationary background. Previous work has demonstrated that there are two phases in the pursuit of a target that moves at constant speed. In the first phase, which consists of the first $100 \mathrm{msec}$ after the eyes start to move, the eye movement is driven entirely by visual inputs. The motion of the target relative to the eye (image motion) provides the input, and eye acceleration provides the output for this phase of pursuit. In the second phase, the negative-feedback configuration of the system comes into play and allows eye velocity to eventually match target velocity. Because of the important role played by negative feedback, it has been common to assume that pursuit would not require the calibration mechanism offered by motor learning. However, Optican et al. (1985) demonstrated the existence of learning in the initial phase of pursuit. We have now used behavioral experiments to analyze the neural implementation of that learning.

Earlier behavioral and neural analyses of pursuit have led to

Received May 30, 1996; revised Aug. 19, 1996; accepted Aug. 22, 1996.

This research was supported by National Institutes of Health Grant EY03878 and by a Dean's/Anthony health science fellowship from the University of California, San Francisco. We thank Gal Cohen, Sascha du Lac, Vincent Ferrera, Mark Kvale, Jennifer Raymond, Rita Venturini, and our reviewers for comments on earlier versions of this manuscript. We especially thank Jennifer Raymond for many helpful discussions during the course of the research and Vincent Ferrera for programming the video board.

Correspondence should be addressed to Maninder Kahlon, Department of Physiology, P.O. Box 0444, UCSF, San Francisco, CA 94143.

Copyright (C) 1996 Society for Neuroscience $0270-6474 / 96 / 167270-14 \$ 05.00 / 0$ three fundamental concepts that are important both for understanding pursuit and for analyzing learning. (1) The initial pursuit response to the onset of target motion represents an open-loop sensory-motor behavior. Therefore, the properties of the visuomotor transformation for pursuit can be probed during this initial response by measuring the relationship between different target motions and the evoked eye accelerations (Lisberger and Westbrook, 1985). (2) The pathways for pursuit are divided into visual motion processing areas, where the signals for pursuit represent image motion without extraretinal influences (image motion coordinates) and areas that perform higher processing, where signals are represented in "directional" coordinates defined by the direction of the desired eye motion. Lesion, electrical stimulation, and recording studies in monkeys imply that primary visual cortex (V1) and the middle temporal area (MT) represent visual inputs for pursuit in image motion coordinates, whereas the medial superior temporal area (MST), the frontal pursuit area (FPA) in the arcuate sulcus, and the dorsolateral pontine nucleus (DLPN) represent signals for pursuit in directional coordinates (Newsome et al., 1985, 1988; Dürsteler et al., 1987; Segraves et al., 1987; May et al., 1988; Dürsteler and Wurtz, 1988; Mustari et al., 1988; MacAvoy et al., 1991; Gottlieb et al., 1994). (3) Pursuit cannot be thought of as a simple visuo-motor reflex and, instead, appears to include a "switch" that allows visual information only selective access, based on the direction of eye movement, to the neural circuitry that generates pursuit (Goldreich et al., 1992; Grasse and Lisberger, 1992; Schwartz and Lisberger, 1994). Thus, the transition from fixation to pursuit involves both closing the switch to allow visual motion inputs to drive eye motion and processing the 
visual motion inputs to determine the desired direction and speed of eye motion.

The goals of our study were to determine the relationship between experience-dependent learning in pursuit and all three of the fundamental concepts outlined above. Once we had demonstrated repeatable learning in the eye acceleration at the onset of pursuit, our primary approach was to study the generalization of learned changes across a variety of sensory and behavioral parameters. Our results demonstrate that learning generalizes well across different target speeds, sizes, and colors but is specific for the combination of the direction of eye and image motion that was used in the training trials. This directional specificity of learning provides new evidence for the existence of the pursuit switch and implies that learning occurs at or beyond the location of the switch in sites where the signals for pursuit are represented not in image motion but, rather, in directional coordinates.

\section{MATERIALS AND METHODS}

Eye movement recordings and behavioral protocol. Experimental methods were similar to those used by Lisberger and Westbrook (1985). Briefly, four rhesus monkeys were trained to fixate a movable spot for water reinforcements. After initial training, each monkey was anesthetized with Isofluorane, and a scleral search coil was implanted on one eye so that eye position could be measured using the magnetic search coil technique (Judge et al., 1980). At the same time, bolts were implanted in the monkey's skull and attached with dental acrylic to a receptacle that was used for head restraint. After the animal had recovered from surgery, the eye coil was calibrated by holding the target stationary at known positions and requiring the monkey to fixate these positions with its head fixed. The target was then moved slowly, and the monkey learned to pursue it smoothly. The eye-coil calibration was repeated before each daily session.

Pursuit targets (PTs). PTs were generated either on an optic bench or by a frame buffer that sent video signals to a monitor. Targets generated from the optic bench were circular spots of light, $0.5^{\circ}$ in diameter, and were moved by servo-controlled mirror galvanometers that reflected them onto the back of a tangent screen $114 \mathrm{~cm}$ from the monkey's eyes. A smaller red spot provided a fixation target (FT) that was used at the beginning of each trial. Targets on the video monitor were generated by a Piranha frame buffer in a $80486 \mathrm{PC}$ and were displayed on a $50 \mathrm{~cm}$ color monitor that was placed $76 \mathrm{~cm}$ from the monkey's eyes. The video system had a noninterlaced refresh rate of $60 \mathrm{~Hz}$ and provided apparent motion with a $16 \mathrm{msec}$ temporal separation between presentations of the target. This stimulus generates pursuit with latencies and accelerations similar to those evoked by the fiber optics targets moved with mirror galvanometers (compare Lisberger and Westbrook, 1985, and Ferrera and Lisberger, 1995). In addition, accelerations evoked by the two stimuli can be compared directly for monkeys A and D in Figures 6 (target motion generated by mirror galvanometers) and 7 (target motion generated by frame buffer). The spatial resolution of the display was 1280 pixels $\times 1024$ lines, and the depth was 8 bits/pixel. The targets were isoluminant red and green squares, 0.3 or $0.75^{\circ}$ in width, and were displayed on a uniform gray background. The FT was a white square, $0.3^{\circ}$ in width. All targets were well above the threshold for detection, and viewing was binocular. Pursuit learning was equally good for targets presented on either the tangent screen or the video monitor. Therefore, we chose the visual presentation method that was best suited to the particular goals of each experiment

Experiment design and learning paradigm. Stimuli were presented in discrete trials that required the monkey to fixate and pursue a target for $\sim 2 \mathrm{sec}$ to obtain a reward. The basic target motion was derived from the step-ramp target motion of Rashbass (1961). In most of the experiments, stimulus presentation followed the sequence illustrated in Figure $1 \mathrm{~A}$. Each trial began when the monkey acquired a FT at straight-ahead gaze. After a random-duration period of fixation $(200-600 \mathrm{msec})$, the fixation light was switched off and a PT appeared $3^{\circ}$ eccentric and immediately began moving toward the fixation point. We used this sequence of target motion when the target was presented on the video monitor or when it was presented using the optic bench and the background was brightly illuminated. In some of our early experiments, we used the target presentation diagrammed in Figure $1 B$. After the monkey had started to look at the FT, there was a random-duration interval (500-800 msec) when both the PT and the FT were on. During this time, the monkey was
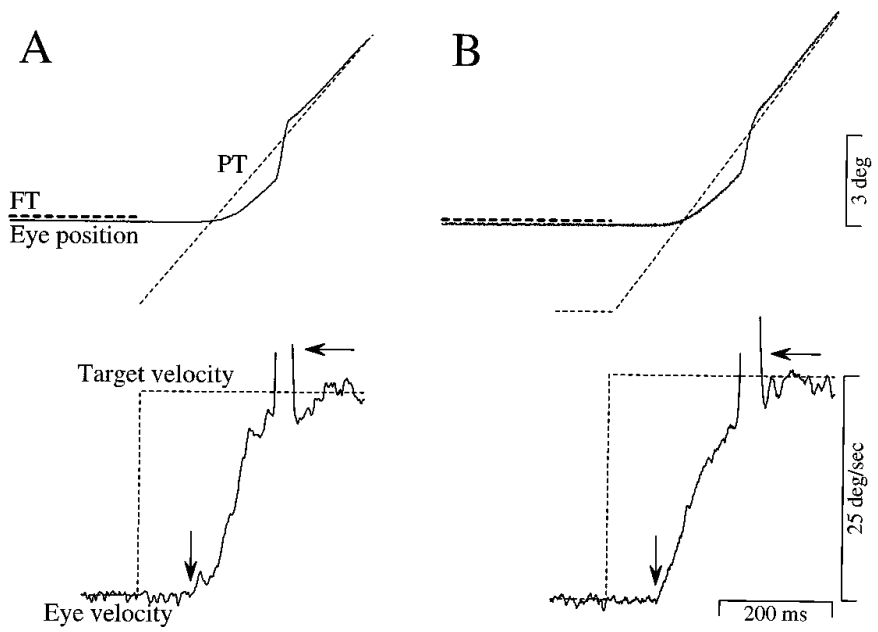

Figure 1. Examples of the two methods of target presentation and the resulting smooth pursuit eye movements. The top traces show eye and target position, and the bottom traces show eye and target velocity as a function of time. Solid and dashed traces show eye and target motion, respectively. The position of the $F T$ is marked by the dark dashed trace, and the position of the $P T$ is marked by the light dashed trace. The actual period during which the FTs and PTs were on and stationary has been truncated in these figures. In the velocity traces, vertical arrows mark the initiation of pursuit, and horizontal arrows mark the rapid deflections of eye velocity associated with saccades. $A$, The PT came on and moved immediately to the right. This example is of target motion on the video monitor. Quantization of target movement attributable to the $60 \mathrm{~Hz}$ frame rate of the monitor resulted in a target velocity of $22.5^{\circ} / \mathrm{sec}$ instead of the desired $25 \%$ sec. $B$, The PT was illuminated but stationary for $800-1100$ msec (truncated). This example is for target motion on the tangent screen so that the target moved at the desired speed of $25^{\circ} / \mathrm{sec}$.

required to ignore the $\mathrm{PT}$, which was stationary at $3^{\circ}$ eccentric, and look at the FT, which was at straight-ahead gaze. The FT was then extinguished, and the PT began to move either toward or away from the FT. This method of target presentation was used when the target was presented with the optic bench and a dimly illuminated background, because the initiation of pursuit is much crisper under these visual conditions if the target is visible and stationary before it starts to move (Krauzlis and Lisberger, 1994). Learning was equally good with either of the two sequences of target appearance and motion shown in Figure $1, A$ and $B$.

Experiments were designed to cause learning that either increased the eye acceleration at the initiation of pursuit evoked by a "test speed" of $10 \% \mathrm{sec}$ or decreased the eye acceleration evoked by a test speed of $25 \%$ sec. Target motion trajectories were of three types. "Test trials" consisted of targets that moved at the test speed for the entire duration of the trial. "Learning trials" consisted of targets that began to move at the test speed but after $100 \mathrm{msec}$ underwent a step increase or decrease in speed (Miles and Kawano, 1986). "Generalization trials" consisted of targets that moved at speeds other than the test speed, were of different color or size, or delivered a brief perturbation of target motion at different times. For each experiment, one direction of motion (left or right) was chosen randomly as the "learning" direction, whereas the opposite direction served as a "control" direction. Each experiment consisted of three periods. In the prelearning period, test trials were intermixed randomly with generalization trials. During the next period, $\sim 300$ learning trials were presented in the learning direction intermixed randomly with 300 test trials in the control direction. The postlearning block of trials was similar to the first block, with a random mixture of test and generalization trials. In the first set of experiments, the velocity generalization series, prelearning and postlearning accelerations were measured from test trials intermixed with generalization trials, whereas in all subsequent experiments, they were measured from learning trials also intermixed with generalization trials. We chose the latter approach to avoid presenting stimuli that would cause unlearning before the learning could be assessed, because at least $75 \%$ of the postlearning block of trials was made up of generalization trials. In both learning and test trials, the monkey was allowed $350 \mathrm{msec}$ after the onset of target motion to bring 
eye position within $2-3^{\circ}$ of the target. Monkeys showed no difficulty in maintaining this fixation requirement for the remainder of each trial.

In experiments designed to cause learned increases in the eye acceleration at the initiation of pursuit, test trials provided target motion at $10 \% \mathrm{sec}$ for the entire duration of the trial, whereas learning trials provided target motion at $10^{\circ} / \mathrm{sec}$ for the first $100 \mathrm{msec}$ and at $30^{\circ} / \mathrm{sec}$ for the remainder of the trial. In experiments designed to cause learned decreases in eye acceleration, test trials provided target motion at $25 \% \mathrm{sec}$ for the entire duration of the trial, whereas learning trials provided target motion at $25 \% \mathrm{sec}$ for the first $100 \mathrm{msec}$ and at $5 \% \mathrm{sec}$ for the remainder of the trial. These values were selected after preliminary experiments showed that they resulted in large changes in eye acceleration. No effort was made to further optimize the stimulus conditions for learning. Usually, we delivered only one set of learning trials a day. On a few days, however, the "learning" and "control" directions were exchanged, and a second learning experiment was begun after allowing the animal at least $30 \mathrm{~min}$ of head-free visual experience. There were no obvious effects of this protocol on the efficacy of the learning paradigm.

Data acquisition and analysis. Experiments were controlled and data were acquired with a laboratory computer. Voltages proportional to eye position were passed through an analog differentiator with a low-pass cutoff at $25 \mathrm{~Hz}(-20 \mathrm{~dB} /$ decade $)$ to obtain eye velocity signals. Comparison of the output of this differentiator with the output from higher-pass digital differentiators with cutoffs at 50 and $100 \mathrm{~Hz}$ revealed that the former minimized noise without affecting latencies or eye accelerations during pursuit. When targets were presented on the tangent screen, target position was monitored by position feedback from the mirror galvanometers that moved the PT. When targets were presented on the video monitor, their position and velocity were computed after the experiment on the presumption that the commands sent to the frame buffer were followed exactly, after correction for the limitations created by the spatial and temporal quantization of the frame buffer. Actual target velocities were 4.5, 9, 22.5, and $29^{\circ} / \mathrm{sec}$ when commands of $5,10,25$, and $30^{\circ} / \mathrm{sec}$, respectively, were sent to the frame buffer. This correction affects only the data shown in Figures 4 and 7. Eye and target position and velocity signals were sampled at $1 \mathrm{kHz}$ and stored for subsequent analysis.

Eye velocity data were analyzed after each experiment on a DEC 3000 computer using an interactive computer program. The eye velocity trace from each trial was displayed on the computer screen, and the initiation of pursuit and the onset of the first saccade were marked with a mousecontrolled cursor. The two eye velocity traces in Figure 1 show typical records, and the downward and leftward arrows show the onset of pursuit and the start of the rapid deflection associated with the first saccade, respectively. If the first saccade occurred within the first $80 \mathrm{msec}$ of pursuit, then the trial was discarded. To obtain low-noise estimates of eye velocity as a function of time, traces were aligned on the initiation of pursuit and averaged over at least 10 trials. To obtain the numbers plotted in all our graphs (except those in Figs. 8, 9), each trial was analyzed individually. The program calculated the difference between eye velocity at the beginning and end of the periods $0-30 \mathrm{msec}$ and 50-80 $\mathrm{msec}$ after pursuit initiation, divided by $30 \mathrm{msec}$ to obtain eye accelerations, and stored these values for subsequent averaging and statistical analysis. Data were sorted according to trial type and direction, and the mean $\pm \mathrm{SD}$ of eye acceleration was calculated for each target motion.

We have elected to analyze only the first $80 \mathrm{msec}$ of pursuit, because it provides a saccade-free "open-loop interval" to probe the visuo-motor transformation for pursuit, uncontaminated by the effects of the external negative feedback loop. This approach has been validated by several previous studies (Lisberger and Westbrook, 1985; Newsome et al., 1988; Krauzlis and Lisberger, 1994) and, in the present study, revealed large changes in the gain of pursuit. By contrast, measurements made later in the trials, after steady-state tracking had been achieved, revealed only that the monkeys were tracking the targets accurately both before and after learning.

\section{RESULTS}

\section{Learned changes in eye acceleration in the open-loop period of pursuit}

Figure 2 illustrates averages of the eye velocities evoked in paradigms used to induce learned increases or decreases in the eye acceleration at the initiation of pursuit. In experiments designed to increase eye acceleration (Fig. $2 A$ ), learning trials presented target speeds (dashed traces) that first stepped to $10 \%$ sec for 100 $\mathrm{msec}$ and then to $30^{\circ} / \mathrm{sec}$ for the rest of the trial. In the first 10 learning trials, the evoked eye speed (thin solid trace) first rose gradually in response to the initial target speed of $10 \% \mathrm{sec}$, then increased to the final target speed of $30^{\circ} / \mathrm{sec}$. In the last 10 of 300 learning trials, the evoked eye speed (thick solid trace) increased more rapidly and reached target speed sooner than it had before learning. The effect of learning on eye speed in the learning trials was evident even in the first $100 \mathrm{msec}$ of pursuit (between the two downward arrows). Because this interval precedes the first visual feedback, the expression of learning cannot result from the negative feedback architecture of the pursuit system and instead must represent a change in the visuo-motor pathways that drive the initiation of pursuit. In Figure $2 C$, comparison of the eye speed from the prelearning (fine trace) and postlearning (thick trace) test trials reveals an increase in the eye speed evoked in the first $100 \mathrm{msec}$ of pursuit (between the two downward arrows) for target motion at a single constant speed. In both the learning and the test trials, eye speed at the end of the trial matched the final target speeds of $30^{\circ} / \mathrm{sec}$ or $10^{\circ} / \mathrm{sec}$ because of the negative feedback configuration of pursuit.

For experiments designed to decrease eye acceleration, the learning trials (Fig. $2 \mathrm{~B}$ ) consisted of target motion at $25 \%$ sec for $100 \mathrm{msec}$ followed by target motion at $5 \% \mathrm{sec}$ for the rest of the trial. In the first 10 learning trials, eye speed (thin solid trace) showed a large initial overshoot that reached a peak at the end of the first $100 \mathrm{msec}$ of pursuit (second downward arrow) and then decreased toward the final target speed of $5 \% \mathrm{sec}$. In the last 10 of 300 learning trials (thick solid trace), the initial rise in eye velocity and the amplitude of the overshoot were both smaller than before learning. Again, the effect of learning can be seen clearly in the first $100 \mathrm{msec}$ of the eye velocity evoked by test trials that presented target motion at $25 \% \mathrm{sec}$ (Fig. $2 \mathrm{D}$ ). In the prelearning tests (thin solid trace), eye speed rose rapidly and settled quickly near target speed. In the postlearning tests (thick solid trace), eye speed rose much more slowly, but negative feedback eventually caused eye speed to reach target speed so that learning had no effect on eye speed at the end of the trials.

Superposition of the eye speeds evoked by learning and test trials (Fig. $2 E, F$ ) provides a direct estimate of the time of the first effects of visual feedback and shows that the expression of learning was very similar in the first $100 \mathrm{msec}$ of the responses to the learning and test trials. In $E$ and $F$, the two traces of the same weight show the responses to learning and test trials either before or after learning. These figure parts make three points. (1) Because each pair of responses to learning and test trials was recorded at a given stage of learning, the two traces should provide comparable probes of the open-loop operation of the pursuit system. The near superposition of the first $100 \mathrm{msec}$ of the eye speed responses for the comparable learning and test trials confirms this expectation. (2) For experiments designed to increase (Fig. 2E) or decrease (Fig. $2 F$ ) eye acceleration, the learning and test trials began to diverge at the time indicated by the second arrow, $200 \mathrm{msec}$ after the onset of target motion. This divergence reflects the difference between the target motions for the learning and test trials, which is the delivery, in the learning trials, of a second step of target speed after $100 \mathrm{msec}$ of target motion. Thus, any eye velocity recorded before the divergence reflects the "open-loop" response of pursuit to the first $100 \mathrm{msec}$ of target motion. (3) Comparison of the eye speed during the open-loop interval in prelearn- 


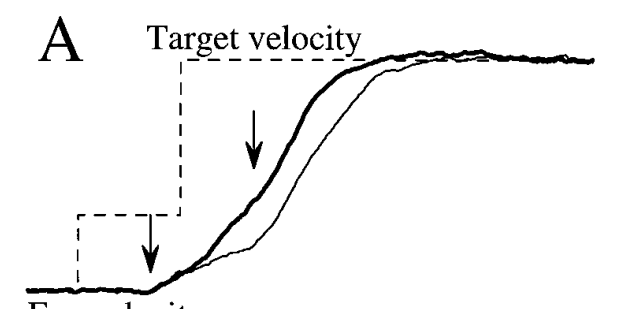

Eye velocity
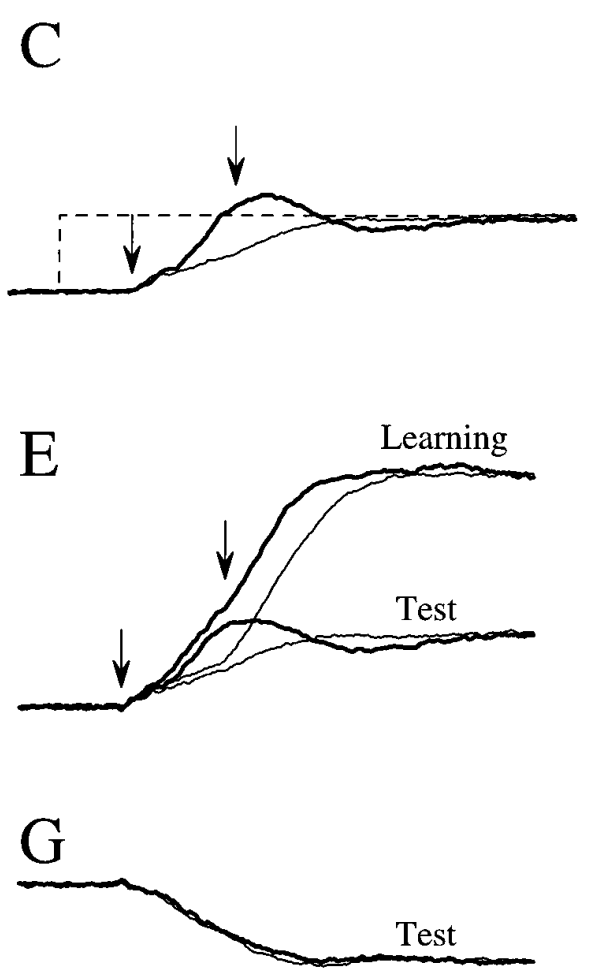
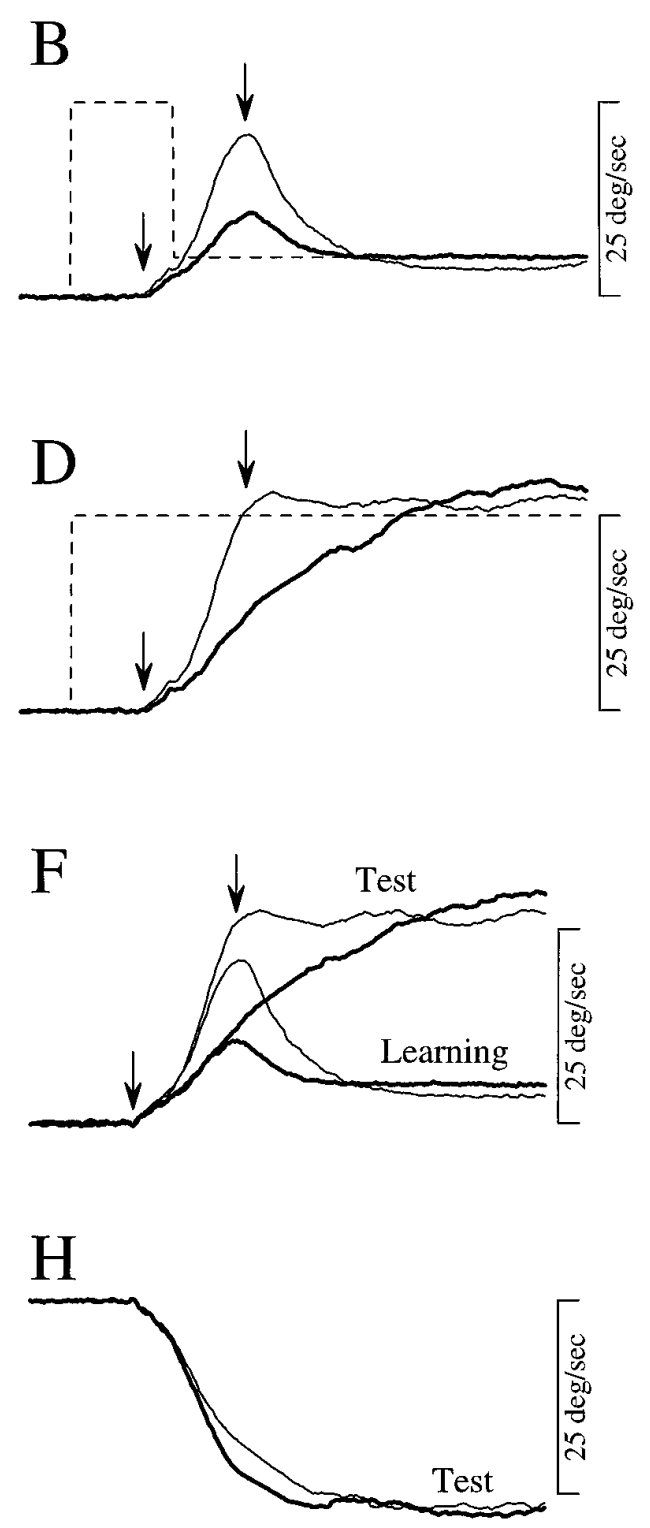

$100 \mathrm{~ms}$
Figure 2. Typical effects of learning on the time course of averaged eye velocity in experiments designed to increase ( $A$, $C, E, G)$ or decrease $(B, D, F, H)$ eye acceleration. In all panels, dashed traces show target velocity, solid traces show eye velocity, fine solid traces show eye velocity before learning, and bold solid traces show eye velocity after learning. Downward arrows delimit the first 100 msec of each response. $A, B$, Fine and bold traces show eye velocity in the first and last 10 learning trials of experiments designed to increase $(A)$ or decrease $(B)$ eye acceleration. $C, D$, Fine and bold traces show eye velocity in the prelearning and postlearning tests for the same experiments illustrated in $A$ and $B . E, F$, Superposition of eye velocity traces for the learning and test trials in $A$ and $C$ and $B$ and $D . G, H$, Fine and bold traces show averages of eye velocity for prelearning and postlearning tests in the control direction. The averages in $A-D$ were aligned on the initiation of target motion, whereas those in $E-H$ were aligned on the initiation of pursuit. Data shown in $A, C, E$, and $G$ are from monkey $\mathrm{F}$, and data shown in $B, D, F$, and $H$ are from monkey $\mathrm{D}$. ing versus postlearning trials demonstrates that the effect of learning in pursuit was expressed clearly before the time of the first visual feedback.

Finally, the averages of eye speed in Figure 2, $G$ and $H$, show examples of the general finding that learning had only a small effect on pursuit in the control direction. For the experiments illustrated in Figure 2, learning trials presented rightward target motion (upward deflections of the traces). The 300 learning trials were intermixed with 300 test trials that presented leftward target motion at one constant speed. The testing speed was $10 \%$ sec and $25^{\circ} / \mathrm{sec}$ in the experiments designed to increase and decrease eye acceleration, respectively. Comparison of the eye speed in the control direction for prelearning (thin traces) and postlearning (thick traces) test trials revealed no discernible change in eye speed in the openloop interval for experiments designed to increase eye acceleration (Fig. 2G) and a small increase for experiments designed to decrease eye acceleration (Fig. $2 H$ ).

\section{Statistical analysis and criteria for data selection}

Table 1 summarizes a statistical analysis of learning for all the experiments we conducted on all four monkeys. The purpose of this analysis was to verify that our learning paradigm produced statistically significant learning in a high percentage of experiments. Therefore, we elected to analyze eye acceleration in the interval 50-80 msec after pursuit initiation, which we will show below is the interval that provided the largest learned changes.

For each experiment, we performed unpaired $t$ tests to compare the values of eye acceleration evoked by a given stimulus before and after learning. In some cases, we compared the responses to target motion in test trials delivered before and after learning, in others, we compared similar responses before and after learning to target motion in learning trials, and when it was available, we compared data from both kinds of trials for the same experiment. It was necessary to use learning trials for the analysis of many experiments, because the prelearning and postlearning blocks of trials included a high percentage of generalization trials (75- 
Table 1. Statistical analysis of the success rate of learning experiments in four monkeys

\begin{tabular}{|c|c|c|c|c|c|}
\hline \multirow[b]{2}{*}{ Monkey } & \multicolumn{2}{|c|}{ Increase acceleration } & \multicolumn{2}{|c|}{ Decrease acceleration } & \multirow{2}{*}{$\begin{array}{l}\text { Control } \\
\text { experiments }\end{array}$} \\
\hline & Test & Learning & Test & Learning & \\
\hline A & $50(6)$ & $55(20)$ & $16(6)$ & $81(6)$ & \\
\hline $\mathrm{D}$ & 91 (12) & $86(7)$ & $100(10)$ & $75(4)$ & $0(4)$ \\
\hline $\mathrm{E}$ & $50(4)$ & $55(9)$ & $100(6)$ & $100(4)$ & $0(6)$ \\
\hline $\mathrm{F}$ & $100(5)$ & $100(5)$ & $100(5)$ & $100(5)$ & \\
\hline Total & 77 (27) & $65(41)$ & $81(27)$ & $86(29)$ & $0(10)$ \\
\hline
\end{tabular}

Each entry in the table reports the percentage of experiments in which the effect of the learning conditions on eye acceleration was significant at the $5 \%$ level or better. Values in parentheses give the number of experiments used for each analysis. Eye acceleration was measured in the interval 50-80 msec after the onset of pursuit and was analyzed separately for learning and test trials. Some experiments appear in the table twice, because both learning and test trials were available for analysis. Data shown as control experiments were taken from the first and last block of trials of experiments that did not deliver learning trials. Instead, monkeys D and E completed 600 test trials that consisted of targets moving rightward or leftward at $10 \mathrm{or} 25^{\circ} / \mathrm{sec}$.

$86 \%)$; the remainder of the trials had to be learning trials instead of standard test trials to avoid running the risk of extinguishing the learning effect that we were trying to measure and analyze. Statistics were usually based on two groups of 10 or more trials, and the minimum numbers of trials were 5 and 10 in the experiments designed to increase and decrease eye acceleration, respectively. We sometimes were forced to use fewer than 10 trials in experiments designed to increase acceleration because of the greater incidence of saccades in the first $80 \mathrm{msec}$ of pursuit.

Table 1 shows that the learning trials caused significant changes $(p \leq 0.05)$ in more than $80 \%$ of experiments designed to decrease eye acceleration and $65 \%$ of experiments designed to increase eye acceleration. The high percentage of statistically significant learning was apparent without regard for whether the measurements were made from test trials or learning trials. All data presented in the remainder of the paper were obtained from experiments that were deemed successful by the above criterion. The rightmost column of Table 1 also shows the absence of any statistically significant changes in initial eye acceleration for five control experiments $(\times 2$ directions of target motion) in which the learning trials were replaced with an equal number of test trials with targets that moved at constant speeds of 25 or $10 \%$ sec. Therefore, we are confident that any significant effects in our data are attributable to the learning paradigm and not to general variability in eye acceleration measurements.

\section{Dynamics and directional specificity of learned changes}

To quantify the dynamics of the learned changes and the effects of learning on eye acceleration in the control direction, we measured eye accelerations separately in intervals from 0 to 30 and from 50 to $80 \mathrm{msec}$ after the onset of pursuit in the learning direction and in the interval from 50 to $80 \mathrm{msec}$ after the onset of pursuit in the control direction before and after learning. The scattergrams in Figure 3 plot each experiment as a separate point and show the mean postlearning eye acceleration as a function of the mean prelearning eye acceleration using different fillings and sizes for each interval and different symbols (circles, squares, diamonds, triangles) for each monkey. Points plot above or below the diagonal line if the postlearning eye acceleration was greater or less than the prelearning eye acceleration, as might be expected for experiments designed to increase or decrease eye acceleration, respectively. Data were included in these graphs only if the analysis summarized in Table 1 revealed a statistically significant effect of learning on eye acceleration in the interval from 50 to 80 msec after the onset of pursuit.

For experiments designed to increase eye acceleration (Fig. $3 A$ ), learning caused the biggest increase in eye acceleration in the interval 50-80 msec after the onset of pursuit in the learning direction (large filled symbols), small increases in eye acceleration in the interval 0-30 msec after the onset of pursuit in the learning direction (open symbols), and little or no change in eye acceleration in the interval $50-80 \mathrm{msec}$ after the onset of pursuit in the control direction (small filled symbols). For experiments designed to decrease eye acceleration (Fig. 3B), learning still had the largest effect on eye acceleration in the interval 50-80 msec after the onset of pursuit in the learning direction (large filled symbols). However, there were also clear and statistically significant $(p<$ 0.05 ) effects in the other analysis intervals. Learning caused a clear decrease in eye acceleration in the interval 0-30 msec after the onset of pursuit in the learning direction (open symbols, paired $t$ test, $\left.t_{(3)}=7.18, p<0.01\right)$ and substantial increases in eye acceleration in the interval $50-80 \mathrm{msec}$ after the onset of pursuit
Figure 3. Summary of the effect of learning on eye acceleration for early $(0-30 \mathrm{msec})$ and late $(50-80 \mathrm{msec}) \mathrm{pe}-$ riods of open-loop pursuit in the learning and control directions. Data are from 43 experiments on four animals that resulted in significant effects $(p<$ $0.05)$ on eye acceleration in the late $(50-80 \mathrm{msec})$ period of the initiation of pursuit in the learning direction and that had 10 or more trials available for averaging in the control direction. Filled symbols indicate values obtained from the late period, and open symbols indicate values obtained from the early period. Large symbols indicate values obtained from the learning direction, and small symbols indicate values obtained from the control direction. Different symbols indicate data from different animals as follows: triangles, monkey $\mathrm{E}$; circles, monkey $\mathrm{D}$; squares, monkey $\mathrm{A}$; diamonds, monkey F.
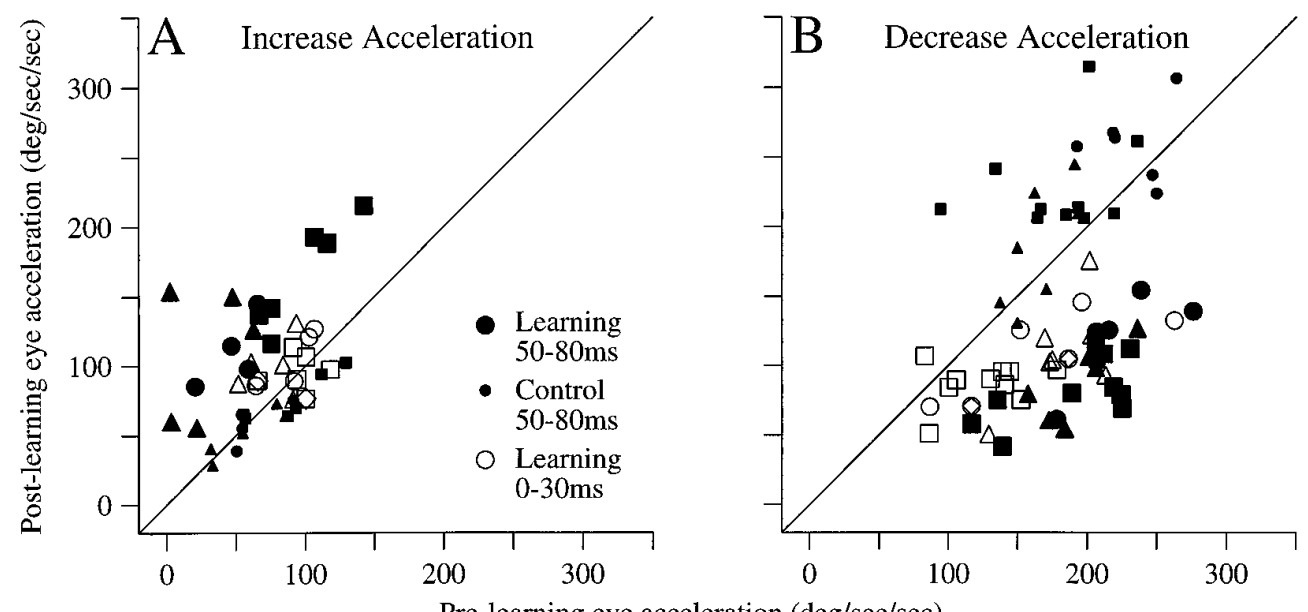
in the control direction (small filled symbols, paired $t$ test, $t_{(3)}=$ $-3.41, p<0.05)$.

Comparison of the distribution of each type of symbol along the $x$-axis in Figure 3 reveals that the prelearning eye accelerations were generally smaller for experiments designed to increase (Fig. $3 A$ ) than for those designed to decrease (Fig. $3 B$ ) eye acceleration. This difference reflects the fact that the testing target speeds were $10 \%$ sec and $25 \%$ sec, respectively, for experiments designed to increase and decrease eye acceleration. Higher target speeds normally evoked larger values of eye acceleration (Lisberger and Westbrook, 1985). It should also be noted that during each experiment, the presentation of target motion at constant speed in the control direction actively biased the system to avoid generalization of learning to the control direction. In this light, it is striking that we found a statistically significant increase in eye acceleration in the control direction in experiments designed to decrease eye acceleration in the learning direction. Finally, it is important to point out that the selection of data, according to the criteria of Table 1, did not bias the results in Figure 3. We did not see any statistically significant effects of learning on eye acceleration in the interval from 0 to $30 \mathrm{msec}$ after the onset of pursuit in experiments that were excluded from Figure 3, because they failed to show statistically significant learning in the interval from 50 to $80 \mathrm{msec}$ after the onset of pursuit.

\section{Time course of learning}

Most of the learning seemed to occur within the first 200 trials, and the time course of learning did not show any consistent changes as experiments were repeated on individual monkeys. Figure 4 shows the time course of learning for 17 experiments on two monkeys. For each experiment, the graphs plot eye acceleration 50-80 msec after the onset of pursuit in learning trials as a function of the number of learning trials the monkey had completed during the experiment. Eye accelerations were averaged from 10 consecutive trials and are plotted as a function of the number of the fifth trial in each group of 10 trials. In each graph, the points connected by the lines with the shortest dashes describe the time course of learning in the first experiment of a given type for each monkey, and the points connected by the lines with the longer dashes describe the time course of learning for the last experiment. To quantify the time course of learning, we averaged the eye accelerations from different experiments for each monkey and direction of learning and fitted an exponential to the averaged data. These exponentials, plotted as dark solid traces without points in Figure 4, had time constants of 240 and 62 trials for experiments designed to increase eye acceleration on monkeys $\mathrm{D}$ (Fig. 4A) and F (Fig. 4B), and 108 and 171 trials for experiments designed to decrease eye acceleration on monkeys D (Fig. $4 C$ ) and F (Fig. 4D).

\section{Retention of learned changes}

In a few experiments, we tested the retention of learned changes in the initial eye acceleration of pursuit by performing a second postlearning test after the monkey had sat in darkness for $30 \mathrm{~min}$ with his head fixed. We then compared the results of the second postlearning test with the results from the prelearning test trials and the first set of postlearning test trials that were completed immediately after learning. The bar graphs in Figure 5 illustrate measurements of eye acceleration in the interval $50-80 \mathrm{msec}$ after the onset of pursuit for a total of four experiments on two

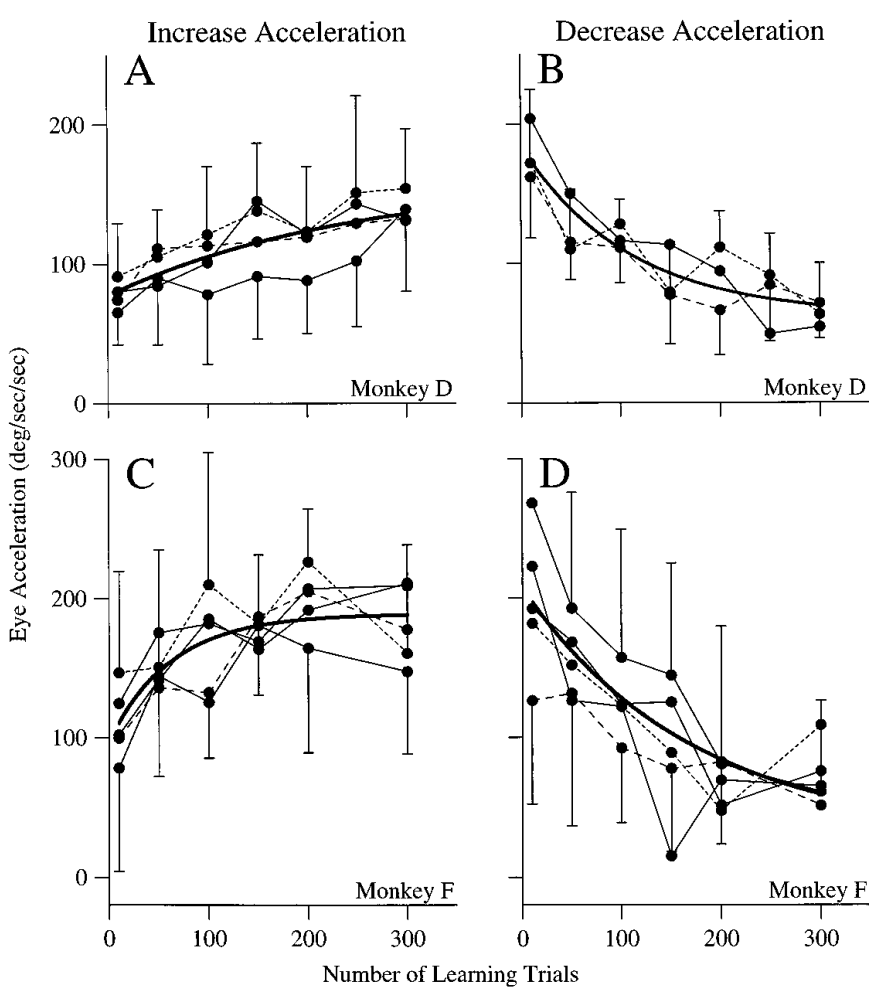

Figure 4. Time course of learning in two animals. Eye acceleration in the $50-80 \mathrm{msec}$ interval was averaged for groups of 10 learning trials and plotted as a function of the number of the fifth trial in the group. $A, C$, Experiments designed to increase eye acceleration. $B, D$, Experiments designed to decrease eye acceleration. For each direction of learning and each animal, points from the first experiment for each animal are connected with small dashes, whereas those from the last experiment are connected with large dashes. The light solid traces connect data from the remaining experiments. The bold curve in each plot is an exponential fit to the mean of data points across all experiments. Error bars indicate $1 \mathrm{SD}$. To simplify the graphs, both leftward and rightward eye accelerations are plotted as positive numbers.

monkeys. All data shown in this figure are taken from test trials in which targets moved at $25 \% \mathrm{sec}$ or $10 \% \mathrm{sec}$, depending on whether the experiment was designed to decrease or increase eye acceleration. Learning trials were not intermixed with the test trials for these experiments. In each case, the second postlearning test yielded eye accelerations similar to those measured in the first postlearning test. A one-factor ANOVA done on each experiment revealed a statistically significant effect of test time (pre-, post-, post-plus-30 min) on eye acceleration. Post hoc tests (Bonferroni/ Dunn) revealed significant differences $(p<0.05)$ between the prelearning test and the first postlearning test as well as between the prelearning test and the second postlearning test in all four experiments. In contrast, the differences between the first and second postlearning test were not statistically significant in any of the four experiments. These results show that learned changes in the eye acceleration at the initiation of pursuit are retained, at least over the $30 \mathrm{~min}$ time period that was required to generate the changes in the first place. We have not investigated the natural decay time constant of these changes further, although we did note that there was usually overnight recovery when the monkeys were allowed natural viewing conditions in the home cage after a learning experiment. 

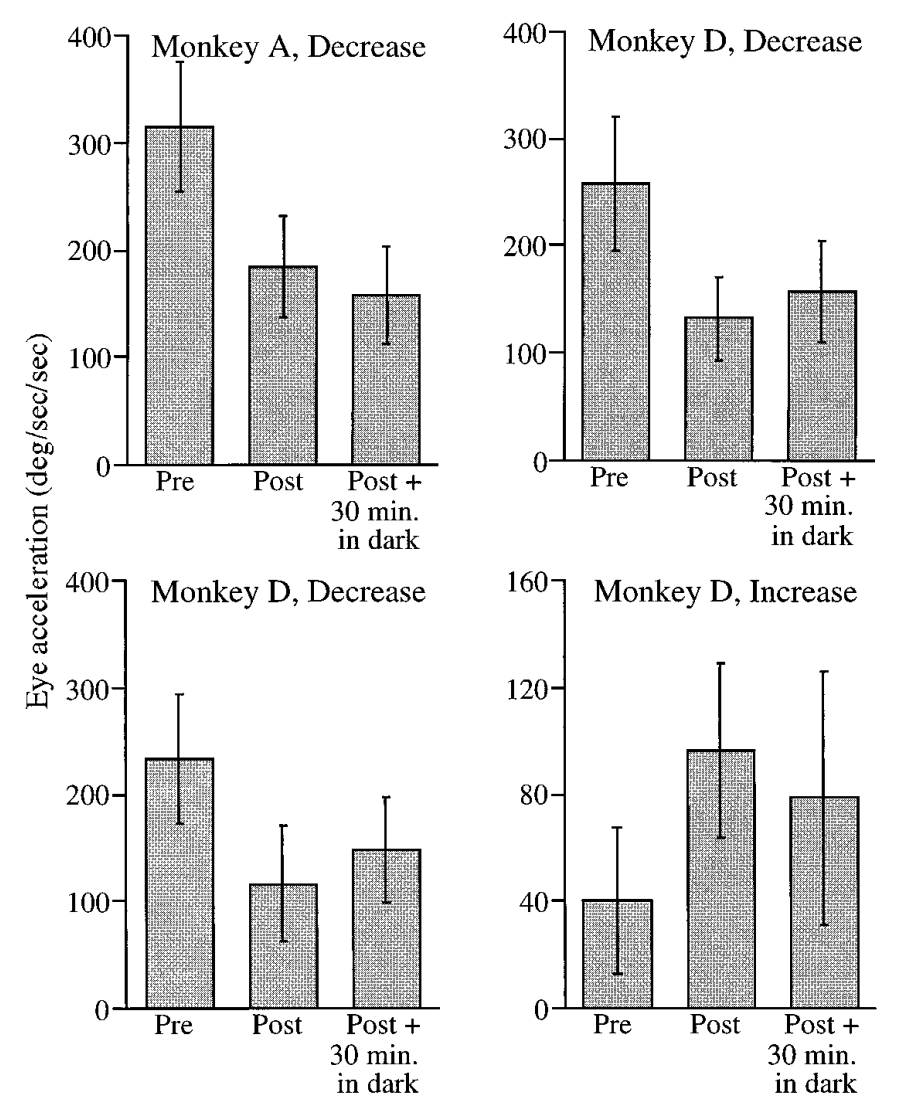

Figure 5. Retention of learned changes after $30 \mathrm{~min}$ in the dark. The four bar graphs show data taken from test trials in four different experiments on two monkeys. Both leftward and rightward accelerations are plotted as positive numbers. Error bars indicate $1 \mathrm{SD}$.

\section{Generalization of learning to different target speeds, colors, and sizes}

Learning generalized well across a range of speeds in experiments designed to increase or decrease initial eye acceleration. To test for generalization across target speeds, the prelearning and postlearning blocks of trials included target motion at single constant speeds ranging from 10 to $45^{\circ} / \mathrm{sec}$ in both the learning and the control direction. Figure 6 shows the results of 22 experiments on three monkeys. Each graph plots eye acceleration in the interval from 50-80 msec after the onset of pursuit as a function of target speed. The three rows of graphs show data from the three monkeys, and graphs on the left and right show results from experiments designed to increase and decrease eye acceleration, respectively. For each set of connected points in Figure 6, the relationship between target speed and eye acceleration was approximately linear over the range of target speeds we used (Lisberger and Westbrook, 1985). Comparison of prelearning (solid lines, filled symbols) and postlearning (dashed lines, open symbols) tests revealed consistent changes in eye acceleration at all testing speeds. In general, learning caused larger absolute changes in eye acceleration for target motion at higher speeds. However, further analysis revealed that learning caused larger percentage changes at lower speeds. Thus, learning-induced changes in the relationship between target speed and eye acceleration could not be described simply as a constant offset or as a multiplicative gain change.

In a separate set of experiments, we found that learning in pursuit generalized as we varied the color or size of the testing targets. In these experiments, target motion was always at the test speed, but PTs in the test trials differed in color and size. Targets were 0.3 or $0.75^{\circ}$ isoluminant red and green squares. Learning was induced with the $0.75^{\circ}$ green square. Figure 7 summarizes the results of 19 experiments in three monkeys in which learning was induced with the $0.75^{\circ}$ green square as a target. The graphs plot the mean eye acceleration in the interval from 50 to $80 \mathrm{msec}$ after the initiation of pursuit as a function of the PT, called "green," "small green," "red," and "small red" for each monkey. Comparison of the means across experiments for data obtained before learning (filled symbols, solid lines) and after learning (open symbols, dashed lines) reveals that the effects of learning generalized from the large green target used in the learning trials (indicated by vertical arrows) to different targets. In the one exception to this general rule, learning did not generalize to the small red target in experiments designed to increase acceleration in monkey $\mathrm{F}$ (Fig. 7, left panel, diamonds). Results consistent with generalization were also obtained in companion experiments using the small red square as the learning target (data not shown).

\section{Context specificity of learning}

We have shown in the preceding sections that learning in pursuit induces changes in the initial eye acceleration of pursuit when a given direction of retinal image motion is used to initiate pursuit from fixation. We now describe the extent to which the learned changes generalize if the same image motion is introduced in different behavioral conditions.

We again used the pursuit learning paradigm described earlier and altered the exact generalization trials used in the prelearning and postlearning tests. Instead of presenting only continuous target motion to test learning, we delivered brief perturbations of target motion under different initial conditions. For example, Figure $8 A$ shows a generalization trial that presented a brief perturbation of target motion during fixation. The trial began with the monkey fixating at straight-ahead gaze. At the time when the target would normally undergo step-ramp motion, it instead stepped to $3^{\circ}$ eccentric and remained stationary. After $500 \mathrm{msec}$, the target underwent a perturbation that consisted of motion to the right at $6 \% \mathrm{sec}$ for $100 \mathrm{msec}$. The perturbation appears as a brief pulse in the target speed trace and a brief ramp in the target position trace (dashed lines). Figure $8 B$ shows a trial in which the same perturbation of target motion was delivered $500 \mathrm{msec}$ after the onset of rightward target motion at $10^{\circ} / \mathrm{sec}$. In this case, the perturbation still appears as a pulse in the target speed trace but caused only a brief increase in the steepness of the target position trace and is therefore more difficult to discern. Comparison of the traces in Figure 8, $A$ and $B$, reveals that the same perturbation was delivered at the same time and for the same target position in both fixation and pursuit trials. The only difference is that the initial conditions were fixation in Figure $8 A$ and rightward pursuit at $10^{\circ} / \mathrm{sec}$ in Figure $8 B$.

The responses to the perturbations were isolated and quantified by comparing averages of eye velocity for target motions that differed only in whether the $100 \mathrm{msec}$ pulse of target velocity was presented. For example, Figure $8 C$ shows the time course of average eye velocity for steady fixation $\left(a^{\prime}\right)$ and for pulses of target velocity presented during fixation $(a)$. Similarly, Figure $8 D$ shows the average eye velocity for pursuit of target motion at $10^{\circ} / \mathrm{sec}\left(b^{\prime}\right)$ and for trials in which pulses were presented $500 \mathrm{msec}$ after the onset of target motion at $10^{\circ} / \mathrm{sec}(b)$. In each case, the solid lines show the average eye velocity, and the dashed lines show target 


\section{Increase Acceleration}
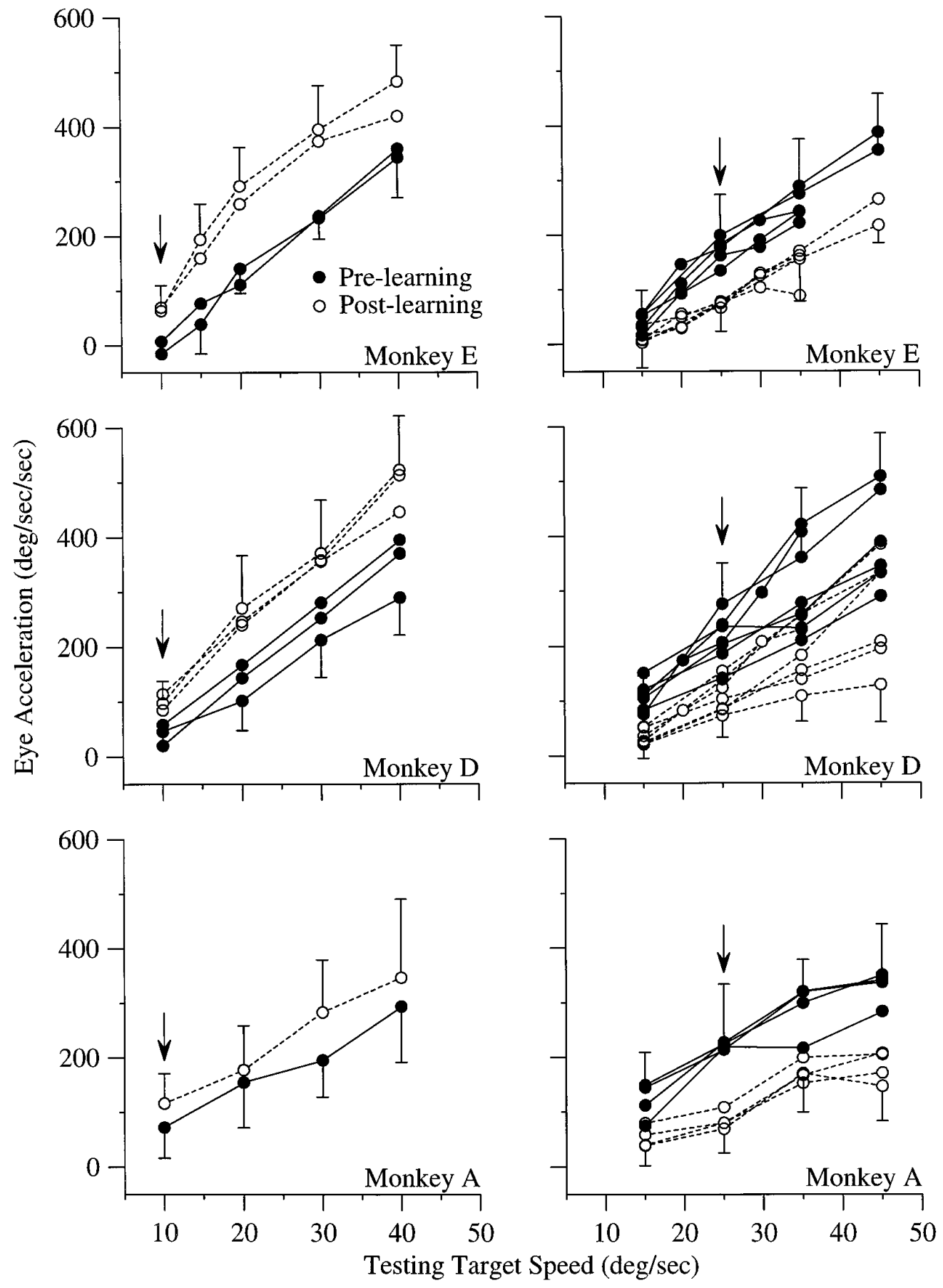

\section{Decrease Acceleration}
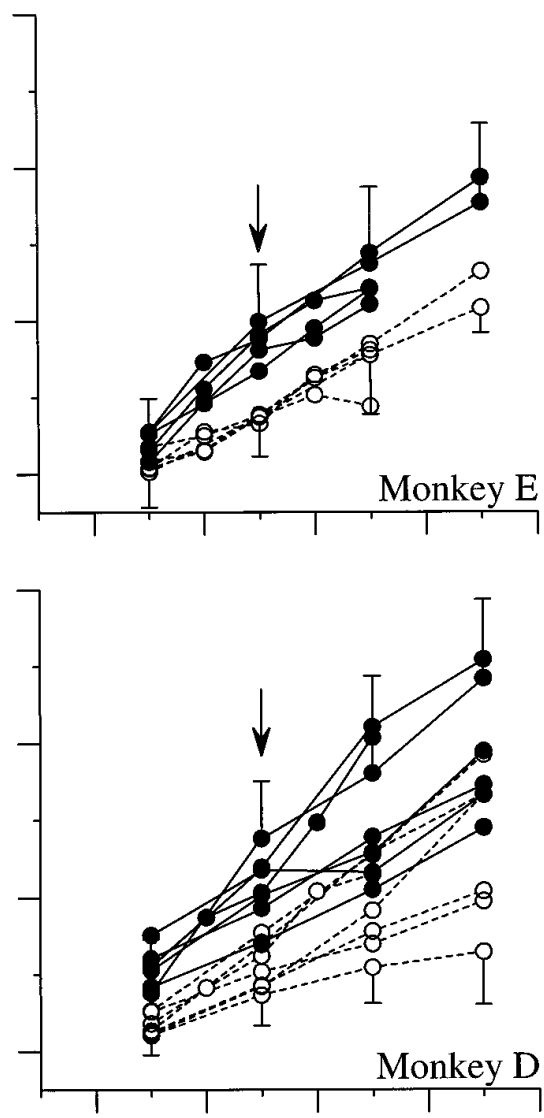

Figure 6. Generalization of learned changes to different target speeds for 22 experiments on three monkeys. Each set of connected points shows the relationship between eye acceleration in the 50-80 msec period and target speed for test trials run either before or after learning. Filled circles connected by solid lines plot data obtained before learning. Open circles connected by dashed lines plot postlearning eye accelerations. The downward arrows indicate the standard test speeds, which were 10 or $25^{\circ} / \mathrm{sec}$ for experiments designed to increase or decrease eye acceleration, respectively. Both leftward and rightward accelerations are plotted as positive numbers. velocity. To evaluate the response to the pulse alone, we subtracted the averaged eye velocity for a given target motion without the pulse from the eye velocity for the same target motion with the pulse. Thus, the difference eye speed traces in Figure $8 E$ show the responses to a $100 \mathrm{msec}$ pulse of target velocity delivered during fixation $\left(a-a^{\prime}\right)$ and during pursuit of target motion at $10^{\circ} / \mathrm{sec}$ $\left(b-b^{\prime}\right)$. These responses are directly comparable, because eye velocity was nearly equal to target velocity at the time each pulse was applied so that the pulses presented during fixation and pursuit provided the same retinal image motion under different initial conditions.
Figure $8, F$ and $G$, shows that learning generalized to brief pulses of image motion presented during pursuit, at least when both pursuit and the image motion provided by the pulses were in the learning direction. The four graphs plot the peak difference eye speed, which was always in the interval from 100 to $200 \mathrm{msec}$ after the onset of the pulse of target speed, as a function of the initial conditions (Fixation or Pursuit) for 21 experiments on two monkeys. Three results are evident from these experiments. (1) The response to the pulse was always larger if the pulse was presented during pursuit than if it was presented during fixation (Schwartz and Lisberger, 1994). This effect was seen in each 
Figure 7. Generalization of learned changes to targets of different colors and sizes in 19 experiments on three monkeys. Each set of connected points plots eye acceleration in the $50-80 \mathrm{msec}$ period for an individual monkey, averaged across multiple experiments. The abscissa indicates the test target, which was a $0.75^{\circ}$ green target (Green), a $0.3^{\circ}$ green target (Small Green), a $0.75^{\circ}$ red target (Red), and a $0.3^{\circ}$ red target (Small Red). As before, the filled symbols connected by a solid line show prelearning accelerations, and the open symbols connected by dashed lines show postlearning eye accelerations. The learning target in these experiments was always the large green target (Green), and accelerations evoked by this target are marked by the vertical arrows. SEs are shown for the data with the greatest variance in each plot. Both leftward and rightward accelerations are plotted as positive numbers. Squares indicate monkey A; circles, monkey D; diamonds, monkey F.
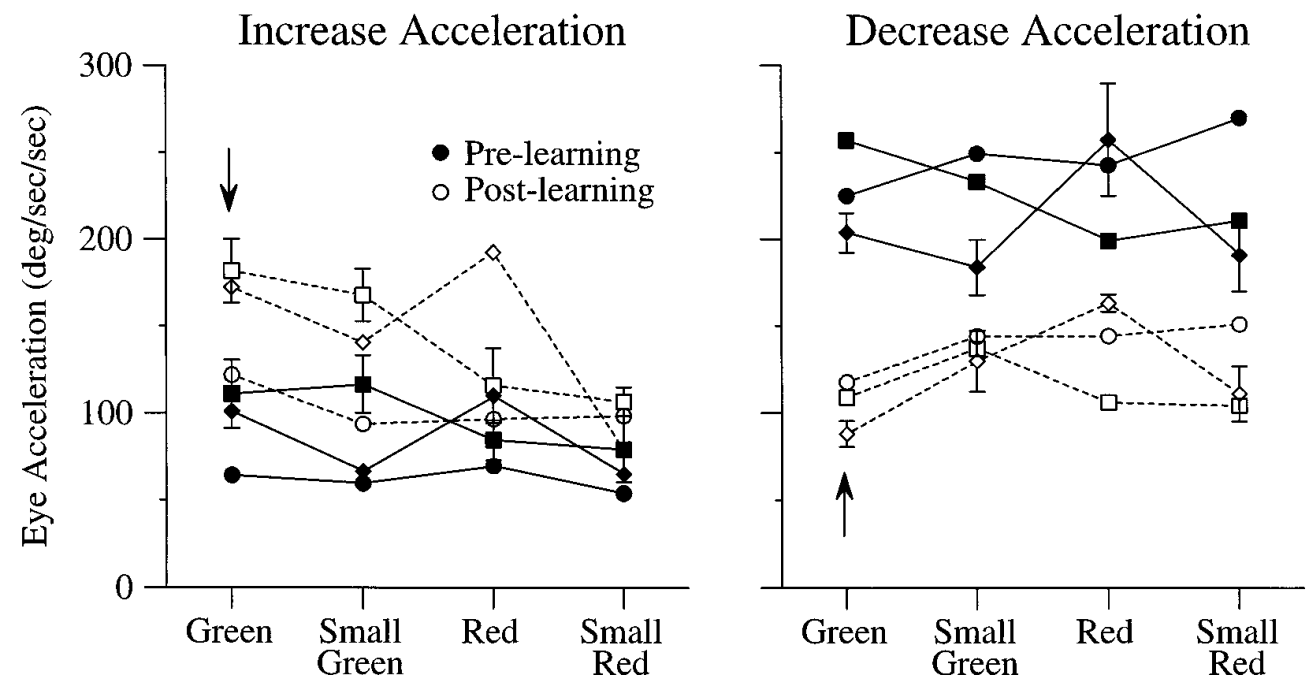

individual experiment (different symbols) as well as in the mean response amplitudes across experiments (lines). (2) The learning generalized to a pulse of image motion that began at the fovea and moved away from it (both columns in each plot), even though image motion started $3^{\circ}$ away from the fovea and moved toward it in the learning trials. Therefore, these results show a generalization of learning over $3^{\circ}$ near the fovea and into the opposite hemifield. (3) The effects of learning appeared without regard for whether the pulse of image velocity was delivered during fixation or during pursuit. Thus, the responses measured after learning (open symbols, dashed lines) were consistently larger or smaller than those measured before learning (filled symbols, solid lines), depending on whether the learning caused an increase or a decrease in the initial eye acceleration of pursuit. Four two-way repeated-measures ANOVAs on data from each monkey and for each learning paradigm revealed no significant interactions between group (fixation vs pursuit) and learning (prelearning vs postlearning) but revealed significant differences in the amplitude of the response to the perturbation both between fixation and pursuit and between prelearning and postlearning data for each ANOVA $(p<0.05)$.

In the same set of experiments, we also found that consistent, statistically significant generalization of the learned changes in pursuit was restricted to the combination of target and image motion in the learning direction. In this more extensive test of generalization, we analyzed the effect of learning on the responses to all four combinations of target motion and image motion in the learning and control directions. Thus, reading target and eye velocity traces in Figure $9 A$ from left to right, the prelearning and postlearning generalization trials delivered rightward pulses during rightward target motion, leftward pulses during rightward target motion, leftward pulses during leftward target motion, and rightward pulses during leftward target motion. The four combinations of target and image motion pulses are summarized by the combinations of arrows below the four sets of traces in Figure $9 A$.

The four graphs in Figure $9 B-E$ plot the peak difference eye speed as a function of the direction of pursuit and image motion pulses (upward arrows indicate the learning direction). Four twoway repeated-measures ANOVAs revealed a significant interaction effect between learning (prelearning vs postlearning) and test condition for all cases. Subsequent pair-wise $F$ tests (Bruning and Kintz, 1987) revealed six statistically significant effects, marked by asterisks in Figure 9 (details of statistical results in figure legend). The first column of each graph in Figure 9 redisplays results from Figure 8 showing that learning was expressed for image motion presented during pursuit if both image motion and pursuit were in the learning direction. In contrast, the last column of each graph shows that there was no evidence of learning in the responses to image motion in the learning direction presented during pursuit in the control direction. The second and third columns show the absence of consistent generalization of learning to the responses to image motion in the control direction, whether presented during pursuit in the learning direction or in the control direction. The two exceptions to the finding that learning was specific to image motion in the learning direction during pursuit in the learning direction were both in experiments designed to decrease acceleration. These exceptions were decreases in response size in monkey $\mathrm{A}$ for the pulse in the control direction during pursuit in the learning direction (Fig. 9C, second column) and increases in response size in monkey $\mathrm{F}$ for the pulse in the control direction during pursuit in the control direction (Fig. 9E, third column).

\section{DISCUSSION}

We have shown that the initial smooth pursuit response to a small moving target is capable of undergoing large learned changes. Although pursuit can use sensory feedback to correct errors on-line, it also uses information about the overall velocity trajectory of a target to change, across multiple trials, the processing in visuo-motor pathways that transform image motion into eye acceleration. The existence of learning in the first $100 \mathrm{msec}$ of the response suggests that the pursuit system attempts to bring eye velocity as close to target velocity as it can before there has been time for feedback. Feedback is used primarily for small, on-line corrections. Thus, the pursuit system seems to use learning as a way to circumvent the problems of control associated with delays in error correction based only on on-line sensory feedback. A similar strategy may be used by other motor systems that normally function with sensory feed- 

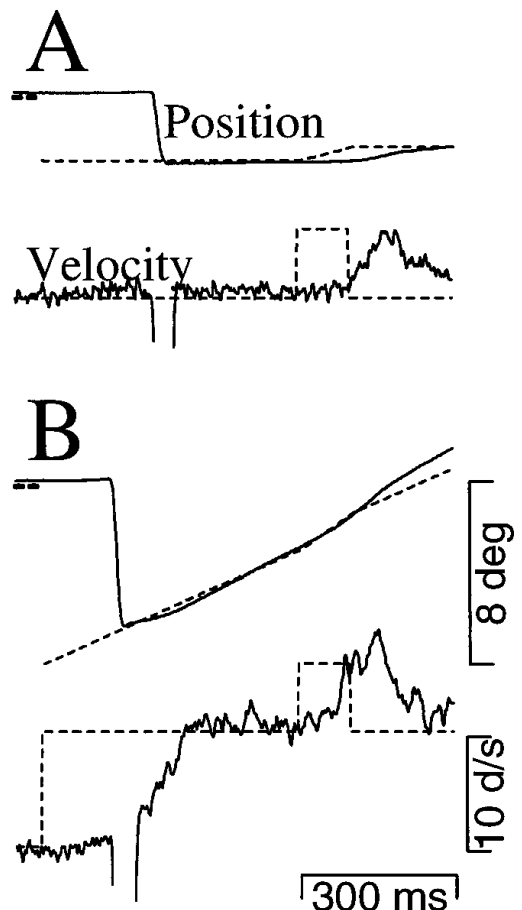

$300 \mathrm{~ms}$

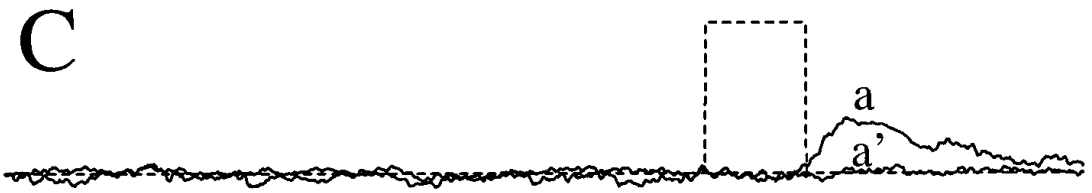

$\mathrm{D}$

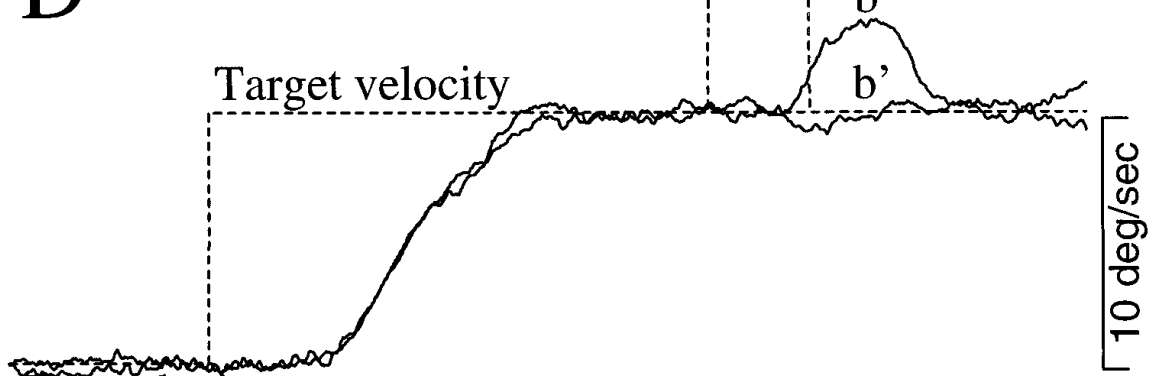
Eye velocity

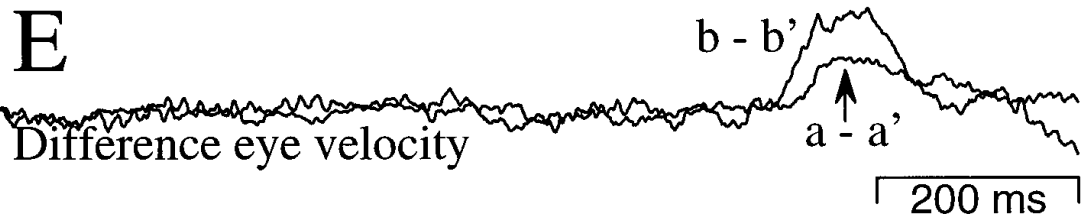

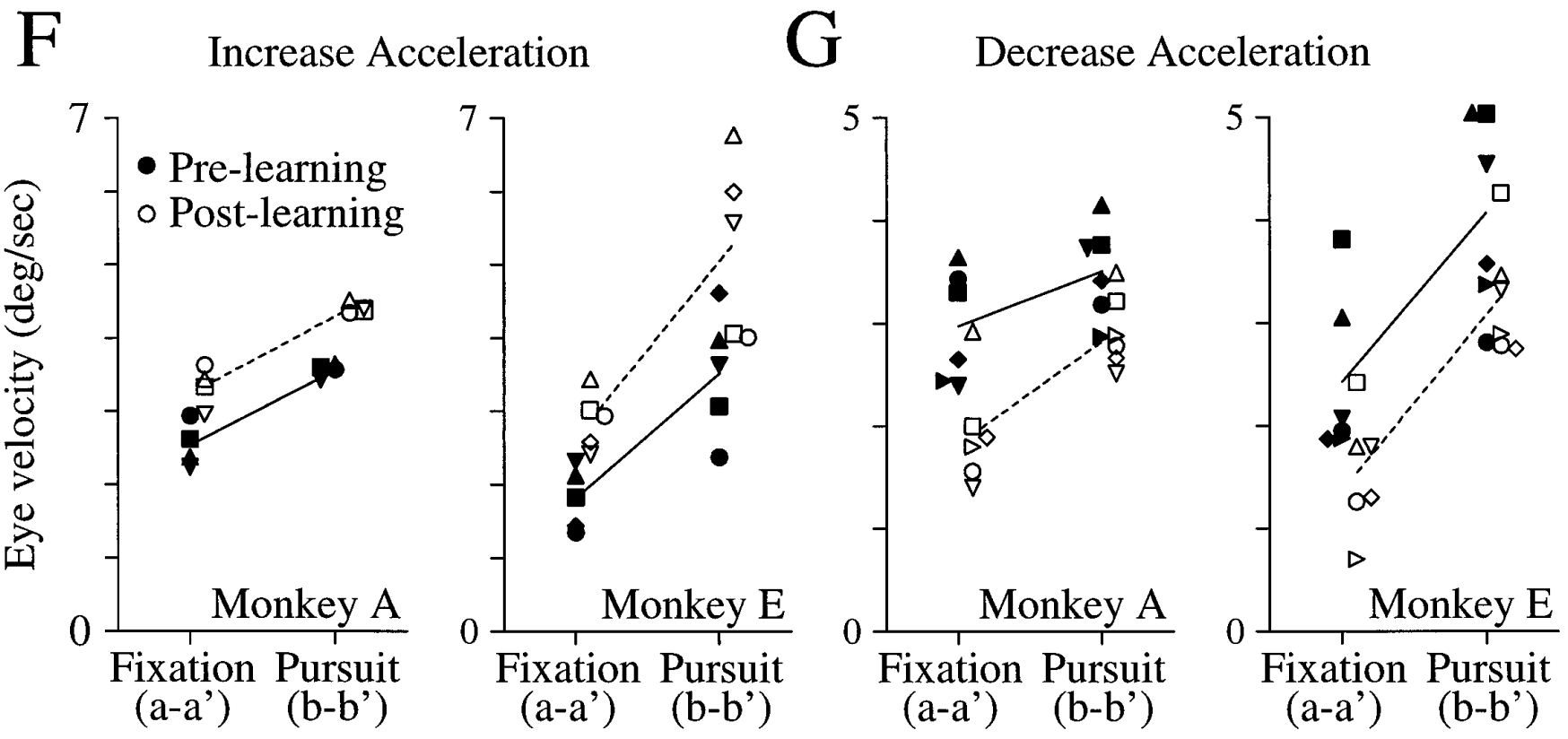

Figure 8. Generalization of learned changes to brief perturbations of target motion during pursuit eye movements. $A-E$ show how the experiment was done and analyzed, and $F$ and $G$ present the results of 21 experiments on two monkeys. In $A-E$, the dashed traces show target motion, and the solid traces show eye motion. $A$, A pulse of target velocity was presented during fixation. $B$, The same pulse of target velocity was presented during pursuit at $10^{\circ} / \mathrm{sec}$. $A, B$, The two bold dashes at the left of the position records in $A$ and $B$ indicate the position of the FT. $C$, The $a$ trace shows the average eye velocity for pulses of target velocity presented during fixation of a stationary target, and the $a^{\prime}$ trace shows the average eye velocity during fixation without a pulse. $D$, The $b$ trace shows the average eye velocity for pulses of target velocity presented during pursuit at $10^{\circ} / \mathrm{sec}$, and the $b^{\prime}$ trace shows the control response to target motion at $10 \% \mathrm{sec}$ without a pulse of target velocity. $E$, Difference eye velocity obtained by subtracting control averages of eye velocity from those obtained in trials that presented pulses of target velocity. The $a-a^{\prime}$ trace shows the response to a pulse of target velocity presented during fixation, and the $b-b^{\prime}$ trace shows the response to a pulse of target velocity presented during pursuit. $C-E$, Each trace is the average of $\sim 10$ eye velocity responses. $F-G$, Plots showing the peak difference eye velocity (from traces like those in $E$ ) before and after learning in experiments designed to increase $(F$ ) or decrease $(G)$ eye acceleration. The two columns of each graph plot the peak difference eye velocity to perturbations of target velocity presented during fixation $\left(a-a^{\prime}\right)$ and pursuit $\left(b-b^{\prime}\right)$. Each symbol represents data from an individual experiment; filled symbols indicate data obtained before learning, and open symbols indicate data collected after learning. Solid and dashed lines show the mean across all experiments before and after learning, respectively. The size of the pulse was $3 \% \mathrm{sec}$ for monkey A and $6^{\circ} / \mathrm{sec}$ for monkey $\mathrm{E}$. 

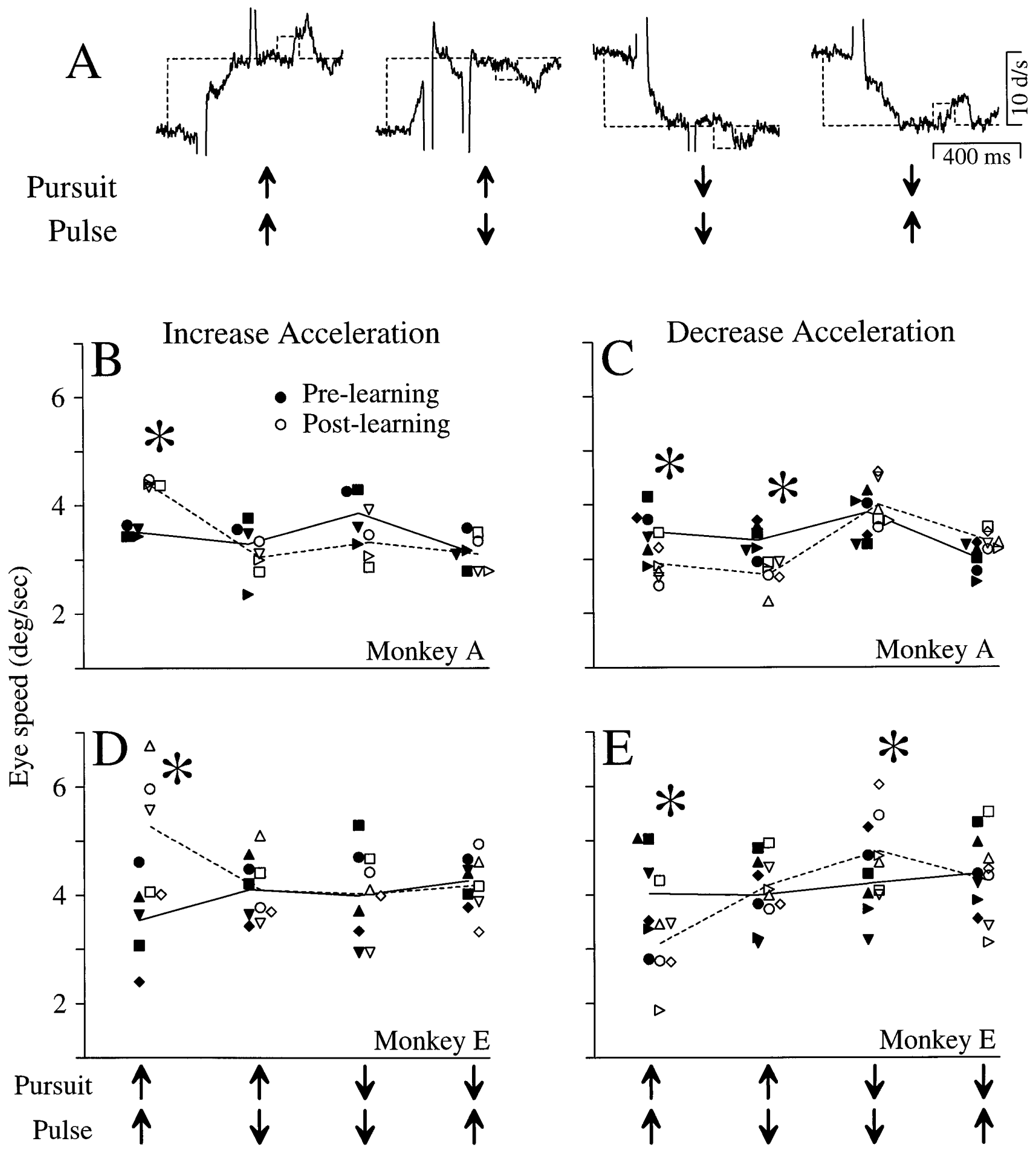

Figure 9. Specificity of learned changes to eye movement and image motion in the learning directions. A, Examples of the trials that presented the four possible combinations of the directions of pursuit and pulses of image motion along the horizontal axis. Dashed traces show target motion, and solid traces show eye velocity. The arrows below the traces summarize pursuit and pulse directions for each test condition, with upward deflections indicating the learning direction. $B-E$, Plots showing the effect of learning on the maximum difference eye speed as a function of the directions of pursuit and image motion. Along the abscissa, the direction of pursuit and image motion is indicated by the arrows. From left to right, each abscissa shows the response to image motion in the learning direction during pursuit in the learning direction; image motion in the control direction, during pursuit in the learning direction, image motion in the control direction during pursuit in the control direction, and image motion in the learning direction during pursuit in the control direction. Data were analyzed as shown in Figure 8. The first column of these graphs contains the same data as the second column of Figure 8. Filled and open symbols show data obtained before and after learning, respectively. Solid and dashed lines plot the means across experiments for data obtained before and after learning, respectively. $B, D$, Experiments designed to increase eye acceleration. $C, E$, Experiments designed to decrease eye acceleration. Large asterisks indicate conditions that showed statistically significant effects of learning. For these conditions, the $p$ values from pair-wise $F$ tests for data in the first column of each graph were $B: F_{(1,12)}=9.58, p<0.01 ; D: F_{(1,16)}=55.99, p<0.001 ; C: F_{(1,20)}=7.97, p<0.02 ; E: F_{(1,20)}=$ 13.00, $p<0.01$; in the second column of $C: F_{(1,20)}=8.98, p<0.01$; in the third column of $E: F_{(1,20)}=5.40, p<0.05$. 
back but must tolerate delays before the feedback is available (Ojakangas and Ebner, 1991).

Our experiments were designed to ensure that the animal did not use a cognitive strategy to modulate the sensorimotor transformation between the speed of image motion and eye acceleration. The direction of pursuit was always randomized and unpredictable, with one direction consisting of learning trials and the opposite consisting of test trials. Because the learning did not generalize to the test trials in the opposite direction, the animal could not have prepared for a learning trial before the target began to move. Additionally, the monkey did not have to generate a strategy to get rewards, because it was allowed ample time to acquire the target after the onset of target motion in each learning trial. It is still possible that the animal initiated a cognitive strategy in the $100 \mathrm{msec}$ between the time the target began to move and the beginning of eye acceleration. Although it is always hard to discount entirely suggestions of strategy learning, there are some reasons to think that the monkeys in our experiments were not using such tactics. First, there were no savings of the learned changes between experiments. Second, studies using a paradigm similar to ours for visually induced saccadic adaptation in humans have reached a consensus that subjects do not use a cognitive strategy to change their saccadic amplitudes (Miller et al., 1981; Deubel et al., 1986; Albano and King, 1989).

The dynamics of the learned changes in the initial pursuit response support the findings of Lisberger and Westbrook (1985) that there are two components of the open-loop interval of pursuit. Their data, which showed that eye acceleration in the earliest $40 \mathrm{msec}$ of pursuit depends less strongly on target velocity than does eye acceleration in the second $40 \mathrm{msec}$ of pursuit, were interpreted as evidence for early and late components of pursuit with separate visual inputs. Similarly, we have shown that the first $30 \mathrm{msec}$ of eye acceleration shows only small effects of learning as compared with the later component of open-loop pursuit. The dynamics of the learned changes could result from either (1) low-pass dynamics or delays in the neural responses at a site of memory, or (2) learning predominantly in neural pathways that generate, selectively, the late component of the initiation of pursuit. A third possibility is that the learning paradigm simply did not provide a strong impetus for changes in the earliest $30 \mathrm{msec}$ of pursuit (see Miles and Kawano, 1986).

Although Figure 3 and Table 1 raise the possibility that there may be a difference in the learning induced by experiments designed to increase versus decrease eye acceleration, we do not think that the data are conclusive. (1) Although we observed statistically significant changes in eye acceleration in the control direction and in the $0-30 \mathrm{msec}$ interval for the learning direction only in experiments designed to decrease eye acceleration, this may be related to the target speeds used to test the learning and not the direction of the required changes. Because the testing target speed was lower in experiments designed to increase eye acceleration, the prelearning eye accelerations were low. The absolute magnitude of any effects simply may not have been big enough relative to the natural variability of initial eye acceleration to achieve statistical significance. (2) Although Table 1 suggests that it was easier to obtain statistically significant learning in experiments designed to decrease eye acceleration, this difference disappears if we exclude experiments in which we tested whether learning generalized to a brief pulse of target speed. The prelearning and postlearning tests for these experiments included generalization trials with targets that moved at $10 \% \mathrm{sec}$, which would have contributed to an extinction of learning in the re- sponse to the test speed of $10^{\circ} / \mathrm{sec}$ in experiments designed to increase eye acceleration, without affecting the response to the test speed of $25^{\circ} / \mathrm{sec}$ in experiments designed to decrease eye acceleration.

\section{Coordinate system and possible sites of learning}

The patterns of generalization in our data suggest that learning occurs in a reference frame that is neither purely sensory nor purely motor. For example, the failure of learning to generalize to conditions that delivered image motion in the learning direction during pursuit in the control direction (Fig. 9) implies that the learned changes in pursuit do not occur in image motion coordinates. Instead, the expression of learning only during fixation or during eye movement in the learning direction implies that the neurons encoding the learned changes are also influenced by eye movement itself. It is interesting to note that saccadic adaptation studied in humans does not seem to occur in image position coordinates and even generalizes to saccades evoked by auditory stimuli (Frens and van Opstal, 1994).

It seems unlikely that learning is in MT. If learning occurred in MT, then we would expect the responses of cells in MT to be modulated by both the direction of eye motion and image motion. However, electrophysiological studies have failed to reveal any significant indication of extraretinal signals related to pursuit in MT (Newsome et al., 1988) (Ferrera VP, Lisberger SG, unpublished observations). If learning occurred in MT, then we might expect learning to be specific for target motion across a small area in the visual hemifield in which the learning stimulus occurred (Maunsell and Van Essen, 1987; Komatsu and Wurtz, 1988). However, we found that learning generalized to the same image motion across $3-6^{\circ}$ and into the opposite hemifield (Fig. 8). Finally, if learning occurred in MT, we would have expected the generalization to other speeds to be more sharply tuned than it is, to reflect the speed tuning of cells in MT (Maunsell and Van Essen, 1983; Rodman and Albright, 1987; Lagae et al., 1993; Cheng et al., 1994).

At the other end of the system, it also seems unlikely that the learned changes occur in the motor coordinates of eye muscles. Many of the premotor neurons and motoneurons have high spontaneous firing rates and are used for both leftward and rightward pursuit eye movements as well as for other kinds of smooth eye movements such as the vestibulo-ocular reflex (VOR). If learning occurred in the brainstem oculomotor regions or the neural integrator, we would predict that learning would generalize quite widely, at least to both directions of horizontal pursuit and also to the VOR. Our data show that learning did not generalize to both directions of horizontal pursuit, and, although we did not test generalization to the VOR, Lisberger (1994) showed that learning in the VOR did not generalize to pursuit eye movements.

The dependence of the expression of learned changes on the direction of both image and eye motion may fit with results from recent behavioral, lesion, and microstimulation studies suggesting that the initiation of pursuit eye movements involves a transition from fixation to pursuit that can be characterized as a directional "switch." In one set of behavioral experiments, Schwartz and Lisberger (1994) demonstrated the existence of the pursuit switch by showing that the size of the eye velocity evoked by a brief perturbation of target motion depended on when the perturbation was delivered. The responses were small if the perturbation was delivered during fixation and much larger if delivered $500 \mathrm{msec}$ after the onset of pursuit at 
$20-30 \%$ sec. Further, responses were small if the direction of the perturbation was orthogonal to ongoing pursuit and large if the perturbation was along the axis of pursuit. Two other behavioral studies (Grasse and Lisberger, 1992; Kiorpes et al., 1996) have suggested that there are separate switches for different directions of pursuit. The most profound example came from two monkeys that were made strabismic early in life and tested as adults (Kiorpes et al., 1996). With monocular viewing, these monkeys had poor pursuit of targets that moved temporalward with respect to the viewing eye and normal pursuit of targets that moved nasalward. If a brief pulse of temporalward target motion was delivered during nasalward pursuit, however, the evoked change in eye velocity was of normal amplitude. The fact that the same temporalward image motion could evoke poor or excellent pursuit depending on the direction of eye movement during which it was introduced implied that the motion was gated separately by switches that were specific for rightward and leftward pursuit and that these monkeys could not close the switch for temporalward pursuit in either eye.

Lesion experiments have suggested that a number of cortical and subcortical components of the pursuit system are not organized in image motion coordinates but instead operate in directional coordinates like the pursuit switch. Unilateral lesions in MST, FPA, and DLPN all result in nonretinotopic ipsiversive directional deficits in pursuit that are clearly not in image motion coordinates (Dürsteler and Wurtz, 1988; May et al., 1988; MacAvoy et al., 1991). Recordings from these areas, and from the floccular complex and the caudal fastigial nucleus of the cerebellum, have revealed "extraretinal" signals that could not be accounted for by only image motion (Mustari et al., 1988; Newsome et al., 1988; Stone and Lisberger, 1990; Fuchs et al., 1994; Gottlieb et al., 1994). Finally, microstimulation in MST or DLPN caused much larger smooth eye velocities if introduced during ongoing pursuit than if introduced during fixation (May et al., 1985; Komatsu and Wurtz, 1989). This last set of experiments suggests that the MST and DLPN are at and/or upstream from the site of gating by the switch.

In our experiments, the selectivity of learned changes for a precise combination of image motion and eye movement direction has two related implications. First, the selectivity of learning for only the combination of image motion and eye movement directions used for the learning trials provides new evidence for the existence of this previously hypothesized pursuit switch and adds to the evidence that the switch is directional. Without a directional switch, learning should have been expressed in the response to the image motion that induced learning, not only during pursuit in the learning direction but also during pursuit in the control direction. Second, the selectivity of learning for the combination of image and eye movement direction establishes that the locus of pursuit learning is either at the site or sites of the switch or in pathways transmitting signals that control the switch. This introduces the intriguing possibility that the neural implementation of the pursuit switch might itself be subject to longer-term plasticity and thus mediate learning in pursuit. It follows that candidate loci for learning should be drawn from those structures that represent pursuit information in directional coordinates and that are candidates as sites for the pursuit switch. This would include areas in which unilateral lesions give directional deficits in pursuit as well as structures that are at or downstream from sites where the responses to microstimulation are affected by the state of the pursuit system. Thus, possible sites for learning in pursuit include MST, FPA, DLPN, and cerebellum.

\section{REFERENCES}

Albano JE, King WM (1989) Rapid adaptation of saccadic amplitude in humans and monkeys. Invest Opthalmol Vis Sci 30:1883-1893.

Bruning JL, Kintz BL (1987) Computational handbook of statistics, Third Ed. Glenview, IL: Scott, Foresman \& Company.

Cheng K, Hasegawa T, Saleem KS, Tanaka K (1994) Comparison of neuronal selectivity for stimulus speed, length, and contrast in the prestriate visual cortical areas V4 and MT of the macaque monkey. J Neurophysiol 71:2269-2280.

Deubel H, Wolf W, Hauske G (1986) Adaptive gain control of saccadic eye movements. Human Neurobiol 5:245-253.

Dürsteler MR, Wurtz RH (1988) Pursuit and optokinetic deficits following chemical lesions of areas MT and MST. J Neurophysiol 60:940-965.

Dürsteler MR, Wurtz RH, Newsome WT (1987) Directional pursuit deficits following lesions of the foveal representation within the superior temporal sulcus of macaque monkey. J Neurophysiol 5: 1262-1287.

Ferrera VP, Lisberger SG (1995) Attention and target selection for smooth pursuit eye movements. J Neurosci 15:7472-7484.

Frens MA, van Opstal AJ (1994) Transfer of short-term adaptation in human saccadic eye movements. Exp Brain Res 100:293-306.

Fuchs AL, Robinson FR, Straube A (1994) Participation of the caudal fastigial nucleus in smooth pursuit eye movements. I. Neuronal activity. J Neurophysiol 72:2714-2728.

Grasse KL, Lisberger SG (1992) Analysis of a naturally occurring asymmetry in vertical smooth pursuit eye movements in a monkey. J Neurophysiol 67:164-179.

Goldreich D, Krauzlis RJ, Lisberger SG (1992) Effect of changing feedback delay on spontaneous oscillations in smooth pursuit eye movements of monkeys. J Neurophysiol 67:625-638.

Gottlieb JP, MacAvoy MG, Bruce CJ (1994) Neural responses related to smooth-pursuit eye movements and their correspondence with electrically elicited smooth eye movements in the primate frontal eye field. J Neurophysiol 72:1634-1653.

Judge SJ, Richmond BJ, Chu FC (1980) Implantation of magnetic search coils for measurement of eye position: an improved method. Vision Res 20:535-538.

Kiorpes L, Walton PJ, O’Keefe LP, Movshon JA, Lisberger SG (1996) Effects of early-onset artificial strabismus on pursuit eye movements and on neuronal responses in area MT of macaque monkeys. J Neurosci, in press.

Komatsu H, Wurtz RH (1988) Relation of cortical areas MT and MST to pursuit eye movements. I. Localization and visual properties of neurons. J Neurophysiol 60:580-603.

Komatsu H, Wurtz RH (1989) Modulation of pursuit eye movements by stimulation of cortical areas MT and MST. J Neurophysiol 62:31-46.

Krauzlis RJ, Lisberger SG (1994) Temporal properties of visual motion signals for the initiation of smooth pursuit eye movements in monkeys. J Neurophysiol 72:150-62.

Lagae L, Raiguel S, Orban GA (1993) Speed and direction selectivity of macaque middle temporal neurons. J Neurophysiol 69:19-39.

Lisberger SG (1994) Neural basis for motor learning in the vestibuloocular reflex of primates. III. Computational and behavioral analysis of the sites of learning. J Neurophysiol 72:974-999.

Lisberger SG, Westbrook LE (1985) Properties of visual inputs that initiate smooth pursuit eye movements in monkeys. J Neurosci 5:1662-1673.

MacAvoy MG, Gottlieb JP, Bruce CJ (1991) Smooth pursuit eye movement representation in the primate frontal eye field. Cereb Cortex 1:95-102.

Maunsell JHR, Van Essen DC (1983) Functional properties of neurons in middle temporal visual area of the macaque monkey. I. Selectivity for stimulus direction, speed, and orientation. J Neurophysiol 49:1127-1147.

Maunsell JHR, Van Essen DC (1987) Topographic organization of the middle temporal visual area in the macaque monkey: representational biases and the relationship to callosal connections and myeloarchitectonic boundaries. J Comp Neurol 266:535-555.

May JG, Keller EL, Crandall WF (1985) Changes in eye velocity during smooth pursuit tracking induced by microstimulation in the dorsolateral pontine nucleus of the macaque. Soc Neurosci Abstr 11:79. 
May JG, Keller EL, Suzuki DA (1988) Smooth pursuit eye movement deficits with chemical lesions in the dorsolateral pontine nucleus of the monkey. J Neurophysiol 59:952-977.

Miles FA, Kawano K (1986) Short-latency ocular following responses of monkey. III. Plasticity. J Neurophysiol 56:1381-1396.

Miller JM, Anstis T, Templeton WB (1981) Saccadic plasticity: parametric adaptive control by retinal feedback. J Exp Psychol [Hum Percept] 7:356-366.

Mustari MJ, Fuchs AF, Wallman J (1988) Response properties of dorsolateral pontine units during smooth pursuit in the rhesus monkey. J Neurophysiol 60:664-86.

Newsome WT, Wurtz RH, Dürsteler MR, Mikami A (1985) Deficits in visual motion processing following ibotenic acid lesions of the middle temporal visual area of the macaque monkey. J Neurosci 5:825-840.

Newsome WT, Wurtz RH, Komatsu H (1988) Relation of cortical areas MT and MST to pursuit eye movements. II. Differentiation of retinal from extraretinal inputs. J Neurophysiol 60:604-620.

Ojakangas CL, Ebner TJ (1991) Scaling of the metrics of visually-guided- arm movements during motor learning in primates. Exp Brain Res $85: 314-323$

Optican LM, Zee DS, Chu FC (1985) Adaptive responses to ocular muscle weakness in human pursuit and saccadic eye movements. J Neurophysiol 54:110-122.

Rashbass C (1961) The relationship between saccadic and smooth tracking eye movements. J Physiol (Lond) 159:326-338.

Rodman HR, Albright TD (1987) Coding of visual stimulus velocity in area MT of the macaque. Vision Res 27:2035-2048.

Schwartz JD, Lisberger SG (1994) Initial tracking conditions modulate the gain of visuo-motor transmission for smooth pursuit eye movements in monkeys. Vis Neurosci 11:411-424.

Segraves MA, Goldberg ME, Deng SY, Bruce CJ, Ungerleider LG, Mishkin M (1987) The role of striate cortex in the guidance of eye movements in the monkey. J Neurosci 7:3040-3058.

Stone LS, Lisberger SG (1990) Visual responses of Purkinje cells in the cerebellar flocculus during smooth-pursuit eye movements in monkeys. I. Simple spikes. J Neurophysiol 63:1241-1261. 